\title{
Off-Pump Coronary Artery Bypass Grafting in Dextrocardia with Situs Inversus Totalis - A Case Report
}

\author{
Johari $\mathrm{R}^{1}$, Garg $\mathrm{M}^{2}$
}

\begin{abstract}
Dextrocardia with situs inversus totalis, is a rare congenital condition. This goes undiagnosed many a times and is coincidentally diagnosed as in our case. Since individuals with this ailment have normal cardiac physiology and function, they live normal lives. We report one cases of dextrocardia (situs inversus totalis) with triple vessel coronary artery disease. To operate on patients with dextrocardia, surgeons need to make a few modifications. These include using the right internal mammary artery as a conduit for the "left" anterior descending artery, surgeons position - sometimes the operating surgeon may need to stand at the left side of the operating table or may have to come on left side for some time for positioning the heart and placing stabilizing device for optimum exposure. Pre-operative surgical difficulties which may be encountered and choice of conduits should be discussed beforehand.
\end{abstract}

Key words: Dextrocardia, surgeons position

\section{INTRODUCTION}

Dextrocardia with situs inversus totalis is a rare congenital abnormality of development involving a left-handed malrotation of the visceral organs (incidence 1:10,000). Many people with situs inversus are unaware of their unusual anatomy until they seek medical attention for unrelated conditions. The incidence of coronary artery disease in this condition is probably similar to that in the general population. Therefore in our clinical practice, we may come across these patients requiring cardiac interventions. By this report we want to highlight the surgical problems and modifications needed to be addressed.

\section{CASE REPORT}

A 68-year-old lady a known case of hypertension, was admitted to our hospital with history of breathlessness and chest pain of three month duration. (NYHA Class-III). Upon admission ECG showed - Infero-lateral ischaemia. Chest X-ray showed dextrocardia, ECHO confirmed - Dextrocardia, LVEF - 40\%$45 \%$, infero-lateral wall hypokinesia. USG - confirmed situs inversus totalis. Hypothyroidism was detected.

Her coronary angiogram revealed triple vessel disease and dextrocardia. The LAD - proximal $80 \%$ stenosis, LCX proximal 90\% mid stenosis, RCA (dominant) $80 \%$ mid and $99 \%$ distal stenosis. CABG was planned.

\section{Dr. Raghav Johari \\ 2. Dr. M. Garg}

\section{Address for correspondence:}

Dr. Raghav Johari

Metro-MAS Heartcare \& Multi Speciality Hospital

Jaipur, Rajasthan, India

E-mail: johari.raghav@gmail.com
Patient was lined up \& induced as our unit protocol except the pulmonary artery catheter was inserted in left internal jugular vein, instead right, internal jugular vein for pulmonary wedg pressure monitoring. Transesophageal echocardiography was done to performed comprehensive examination of the heart to know about any associated congenital anomalies, which was not found.

Chest was opened through median sternotomy approach. RIMA and 2 lengths of long saphanous vein were harvested. RIMA was found to be of sub-optimal quality, so one more additional length of vein was harvested from other leg.

Heart was stabilized with tissue stabilizing device and vein was anastomosed to LAD. After releasing the heart, pericardial stay sutures which are normally taken on the left side were put on the right side. Surgeon came on the left side and stabilized the heart for OM proximal obtuse marginal. Then grafting was done from the right side. In similar fashion PDA(Posterior Desending Artery) was grafted. Proximal anastomoses were done from the right side. All anastamosis were done with $7 / 0$ proline non cutting needle. Intra and post operative period was uneventful. Total surgical time was 210 mins. Patient required minimal ionotropic supports. She was discharged on the $7^{\text {th }}$ day.

\section{DISCUSSION}

Performing CABG in such patients with dextrocardia is more difficult and challenging as it is not routinely performed. The LAD lies on anterior surface of the heart but more on the right side, hence doesn't cause much of a technical problem in grafting, but choice of conduit must be made beforehand ${ }^{1-3}$. As LIMA(Left Internal Mamary Artery) will fall short, and RIMA(Right Internal Mamary artery) may have to be lengthened. For the PDA and OM which lie on inferior and lateral surface of the heart. The orientation is a problem and heart is lifted from the right side and placing of the tissue stabilizing device is also tricky and pressures have to be 
washed $^{2,3,4}$. Right handed surgeons, who are accustomed of grafting from the right side may find it uncomfortable grafting from the left side.

\section{REFERENCES}

1. Chakravarthy M, Jawali V, Nijagal D. Off pump coronary artery bypass surgery in dextrocardia: A report of two cases. Ann Thorac Cardiovasc Surg 2008;14: 187-191.

2. Ennker IC, Pietrowski D, Ennker J. Off pump myocardial revascularization in an octogenarian patient with dextrocardia and situs inversus. Cardiovasc J S Afr 2006;17:257-258.

3. Kuwata T, Kawata T, Ueda T, Sakaguchi H, Nagasaka S, Taniguchi S. Off pump quintuple coronary artery bypass grafting for situs inversus totalis. Jpn J Thorac Cardiovasc Surg 2004;52:473-475.

4. Bonanomi G, Kostov D, Zenati MA. Emergent off-pump complete myocardial revascularization in dextrocardia. J Cardiovasc Surg 2004; 45: 31- 33. 